\title{
PO01 - Assessment of quality of life of children and adolescents with different types of cancer in all phases of treatment and its completion
}

Efrosini Vlahioti (Greece) ${ }^{1}$; Pantelis Perdikaris (Greece) ${ }^{1}$;

Vasiliki Matziou (Greece) ${ }^{1}$

${ }^{1}$ Faculty of Nursing, National \& Kapodestrian University of Athens

Theme: Accreditation and quality improvement.

Keywords: Adolescents, cancer, children, quality of life.

Aim: The aim of this study was to evaluate quality of life of children and adolescents with different types of cancer in all phases of treatment and its completion.

Patients and methods: The sample of the study was 112 children and adolescents aged 8-20 years. Of these, 56 were new patients with any type of cancer who were receiving treatment and 56 were survivors who had successfully completed at least five years before treatment protocols for childhood cancer.

Results: For children and adolescents, new patients with any type of cancer, during the first measurement at baseline score on the scale for quality of life was 3.44 and 3.88, respectively, in the middle of treatment 3.36 and 3.89 , respectively, and with the completion of treatment 3.43 and 3.89 respectively.

Conclusions: The quality of life of the studied population was not significantly changed during the treatment and after its completion.

\section{PO02 - Clinical profile of children admitted to a paediatric intensive care unit due to acute clinical deterioration}

Claus Sixtus Jensen (Denmark) ${ }^{1,2} ;$ Hanne Aagaard (Denmark); Hanne Vebert Olesen (Denmark) 3; Hans Kirkegaard (Denmark) ${ }^{1}$

${ }^{1}$ Research Center for Emergency Medicine, Aarhus University Hospital; ${ }^{2}$ Aarhus University; ${ }^{3}$ Department of paediatrics, Aarhus University Hospital

Theme: Intensive care

Keywords: Critical care, critical illness, clinical deterioration, PICU

Background: There has been an increased number of critically ill patients admitted to paediatric departments. Only a few studies have described the various causes of unplanned admission to paediatric intensive care units (PICU) due to clinical deterioration. However, an understanding of the nature of illness is important to patient safety.

Purpose: This study described the frequency, clinical characteristics and outcomes of children who experience unplanned admission to a PICU due to clinical deterioration. 
Methods: A registry retrospective descriptive study describing and exploring life-threatening situations leading to unexpected transfers to PICU in hospitalised children. The study includes all paediatric departments in the Central Denmark Region.

Perspective: This study will provide knowledge to assist the research efforts to identify and improve the management of critical ill children in paediatric wards.

\title{
PO03 - Experience from a group of adolescents with severe allergy asthma treatment with omalizumab
}

\author{
Anne Marie Bro Hofbauer (Denmark) ${ }^{1}$; Lone Agertoft (Denmark) ${ }^{1}$ \\ ${ }^{1}$ Hans Christian Andersen Children's Hospital. Odense University Hospital
}

Theme: Complex health care and chronic disease management.

Keywords: Adolescents, allergic asthma, education, omalizumab.

Background: Evidence supports that group educational interventions improve selfperception, self-confidence and attack management in asthma care.

Aim: To evaluate education and structured dialogue in enhancing knowledge and insight in the asthma disease, treatment with omalizumab and social behaviour.

Method: A group of four 13-16-year-olds received education using question and answer cards, and were informed about the effects and adverse effects of omalizumab. The dialogue included life before and after, relation to family and friends, ability and lack of ability to perform physical activities, and dreams of the future. One week later there was a telephone interview.

Results: All four adolescents experienced a positive change in everyday life and ability to manage the asthma disease. Furthermore, they all experienced an enhanced physical ability. In the interview they reflected on their own disease and physical ability.

Conclusion: Adolescents with a chronic disease benefit from sharing thoughts, emotions and knowledge about their disease and treatment.

\section{P004 - Metasynthesis approach in paediatric nursing literature: a scoping review}

Joana Romeiro (Portugal) ${ }^{1}$; Sílvia Caldeira (Portugal) ${ }^{1}$
${ }^{1}$ Instituto de Ciências da Saúde, Universidade Católica Portuguesa

Theme: Professional issues

Keywords: Child, family, metasynthesis, paediatric care, qualitative studies.

Introduction: Metasynthesis is defined as the synthesis of qualitative research. Despite the growing interest in this approach in adult nursing literature, it is not clear how it has been used in paediatric nursing literature. 